\title{
TITLE:
}

\section{The Tensile Strength of Rubber and Rubber Molecule}

\author{
$\operatorname{AUTHOR}(\mathrm{S})$ :
}

Park, Chullchai; Yoshida, Usaburo

\section{CITATION:}

Park, Chullchai ... [et al]. The Tensile Strength of Rubber and Rubber Molecule. Memoirs of the College of Science, Kyoto Imperial University. Series A 1944, 24(2): 109-116

\section{ISSUE DATE:}

1944-03-20

URL:

http://hdl.handle.net/2433/257296

RIGHT: 


\title{
The Tensile Strength of Rubber and Rubber Molecule
}

\author{
By \\ Chullchai Park (朴哲在) and Usaburo Yoshida (吉田卯三郎)
}

(Received, Nov. 28, 1942.)

\begin{abstract}
The tensile strengths of crystallized crude rubber and vulcanizcd rubber are remarkably different from each other at room temperature, but are found to be almost the same at the temperature of liquid air. By assuming that the tensile strength of the crystallized rubber at this low temperature is entirely due to that of its chain molecules the force needed to break a chain molecule of rubber at its weakest point is estimated.
\end{abstract}

The writers prepared ribbons of smoked sheet rubber and vulcanized rubber, each of which was $0.5 \mathrm{~cm}$ wide, $0.3 \mathrm{~cm}$ thick and 6 $\mathrm{cm}$ long. After marking two points $\mathrm{I} \mathrm{cm}$ apart on the surface of a ribbon, it was stretched quickly at room temperature by applying a load of about $700 \%$ stretching degree in the case of smoked sheet rubber and of about $400 \%$ stretching degree in the case of vulcanized rubber. Under these sufficient clegrees of stretching the samples are presumed to be considerably crystallized and to be mostly relieved of intermolecular slipping by stretching. . The stretched specimen was then broken at various temperatures by increasing the load, and its tensile strength was obtained from the value of the breaking load and the cross-sectional area at the instant of the break. The latter was calculated from the values of the cross-sectional area before stretching and the changes of length and density which were caused in stretching up to the final break. The actual experiment was carried on by using the apparatus as shown in Fig. I. In this figure $V$ is a vessel fixed firmly to the heavy table $T$, and $S$ is the test piece of rubber which is to be stretched and broken by applying a load $\mathrm{W}$ over the pulley $\mathrm{P}$. The tensile test was made at various temperatures ranging from $78^{\circ} \mathrm{C}$ to the liquid air temperature. After the specimen was stretched sufficiently at room temperature, it was heated or cooled by pouring a proper liquid or a liquid mixture kept at desired temperatures. Water was used for the temperatures higher than about 
Fig. I .

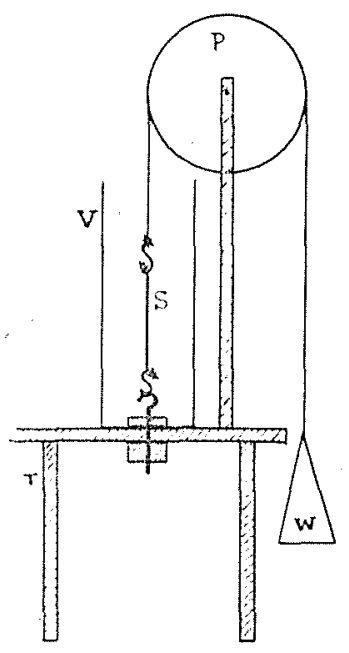

$10^{\circ} \mathrm{C}$, and the liquid air at $-185^{\circ} \mathrm{C}$; and for intermediate temperatures acetone or alcohol mixed with dry ice were employed. Two different kinds of the vulcanized rubber were tested: viz. that of accelerator mixture and that of carbon mixture.

As a control the smoked sheet rubber and the vulcanized rubber were broken suddenly in the same manner as above by heating and cooling at various temperatures without any previous stretching. As stated in the previous papers, ${ }^{1}$ when unstretched rubber is cooled suddenly at low temperatures it undergoes almost no crystallization "and is hardened as an amorphous solid in "super-cooled state. Consequently, by virtue? of the predomi-

Fig. 2

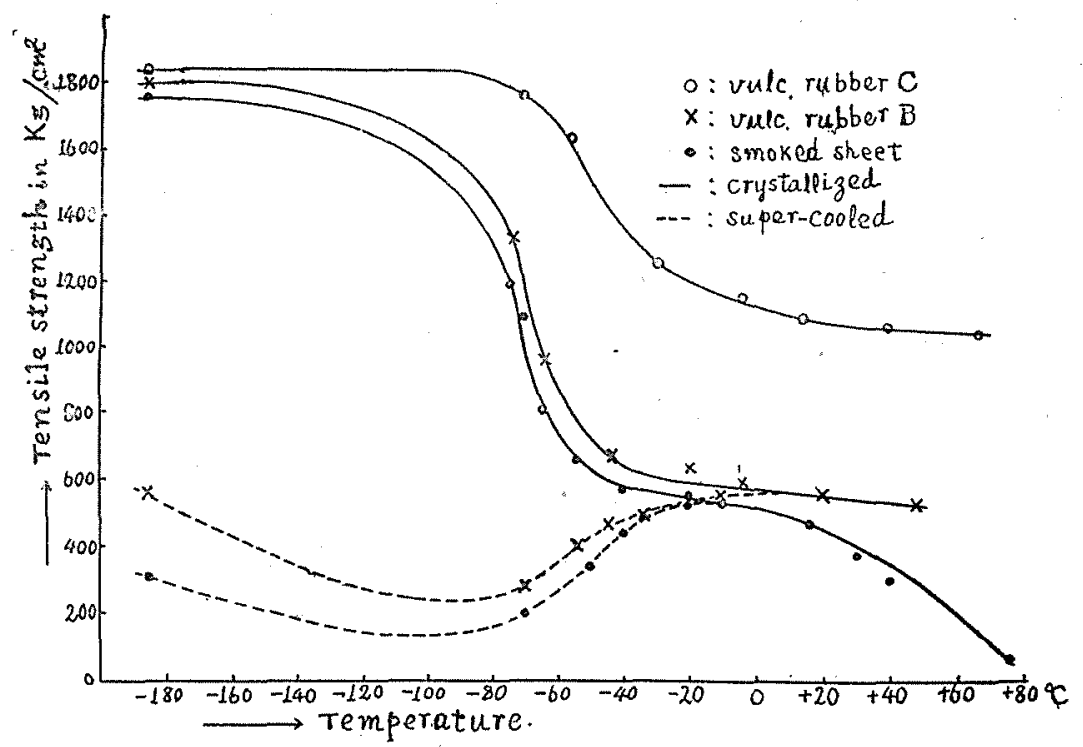

nant intermolecular slipping, a considerable low value of the tensile strength is expected in this case when compared with the specimen cooled in stretched state. The results obtained by the present experiment are shown in Fig. 2, by taking the temperature as abscissa

x. Ch. Park: These Mcmoirs, 22, 259 (1939), 23, 137 (1940): 
and the tensile strength as ordinate. In this figure the curves drawn in full line refer to the specimens broken by being cooled or heated in sufficiently stretched state, those drawn in broken line refer to the specimens broken by being cooled or heated suddenly in unstretched state; and the dots, crosses and smail circles represent respectively the tensile strengths of smoked sheet rubber, vulcanized rubber of accelerator mixture $B$ and the vulcanized rubber of carbon mixture C. As is seen in the figure the tensile strengths of the smoked sheet rubber and the vulcanized rubber which are cooled or heated in sufficiently stretched state increase generally with decrease of the temperature. They are generally very different from each other, being considerabiy higher with the vulcanized rubber $C$, but they converge to almost the same value at the liquid air temperature. This point is especially noteworthy as will be described later. As to the smoked sheet rubber and the vulcanized rubber $B$, which are broken by being cooled suddenly in unstretched state, their tensile strengths behave entirciy differently from the specimens kept stretched at low temperatures. As is expected, the tensile strengths decrease in this case, in contrast to the stretched specimens, with decrease of the temperature from about $-20^{\circ} \mathrm{C}$ to about $-70^{\circ} \mathrm{C}$, and then increase slightly at the temperature of liquid air.

As to the ultimate mechanism of the breaking of the rubber ribbon by stretching two phenomena are conceivable: the mutual slipping between neighbouring chain molecules and the tearing of the chain molecules themselves at the weakest localities in their chain structure. When a ribbon of smoked sheet rubber is stretched very slowly at room temperature, it becomes gradually thinner at some portion without being crystallized noticeably and then undergoes the final rupture. As the elongation of the broken pieces is not much recovered on heating, the rupture of the rubber ribbon in this case seems to be mainly caused by the intermolecular slipping. However, in the present experiment, the rubber ribbons wore broken by suddenly increasing the stretching load by being cooled in a strongly stretched or entirely unstretched state, and the rupture took place with a right section as if the ribbon. was cut with a sharp knife, without becoming gradually thinner as is stated above. Thus by the rupture in this case, the tearing of the long chain molecules is presumable to have happened together with the inter-molecular slipping, though the proportion of these two factors will be different according to the conditions to which the test pieces are subjected. 
Whether or not the rupture of the rubber ribbon in a certain case is mostly due to the tearing of the long chain molecules will be judged by knowing under what condition the inter-molecular slipping is hard to take place. Fig. 3, which has been once shown in a previous paper, ${ }^{1}$ represents the permanent elongation of the smoked sheet rubber ribbons caused by the intermolecular slipping when the ribbons are kept stretched for different durations of time at room temperature. As is seen from the figure, the inter-molecular slipping decteases generally as the degree of crystallization increases by sufficient stretching;

Fig. 3

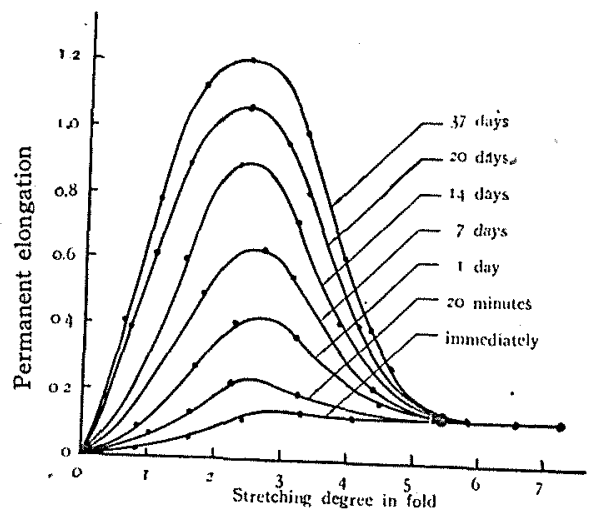
and with extreme stretching to greater than about 5.5 times the original length, no trace of the inter-molecular slipping can be.detected even with the specimen kept for 37 days in the stretched state at room temperature, except a small permanent elongation caused by the process of the initial sudden stretching. On the contrary, when the crystallization is not predominant under insufficient stretching a noticeable inter-molecular slipping, which is roughly proportional to the stretching stress and the amount of uncrystallized part, proceeds gradually during the time the specimen is kept stretched. Further it is repotted in the previous paper that the inter-molecular slipping is remarkable at high temperatures and decreases with decrease of the temperature. Consequently it may be infered from these facts that when an extremely stretched rubber ribbon is cooled to the temperature of dry ice or of liquid air the inter-molecular slipping becomes very difficult, and the tearing of the long chain molecules of rubber at the localities of the weakest interatomic cohesion becomes the main cause for the rupture by stretching of the specimen.

The measurements of the permanent elongations of the vulcanized rubber ribbons of carbon mixture caused by the inter-molecular slipping in a stretched state were made. The rubber ribbons were kept stretched for various durations in three different temperature ranges of $12^{\circ}-18^{\circ} \mathrm{C}, 30^{\circ}-40^{\circ} \mathrm{C}$ and $50^{\circ}-60^{\circ} \mathrm{C}$. The permanent elongations of

I. Ch. Park: These Memoirs, 23, 137 (1940). 
the specimens were obtained as before by putting them in boiling water for a short time after releasing of the stretching tension so that they could completely contract, and then by measuring the amounts of elongation per original unit length. The results of measurement are shown in Figs. 4a, $4 \mathrm{~b}$ and $4 \mathrm{c}$. If we compare these figures with Fig.

Fig. $4 a$

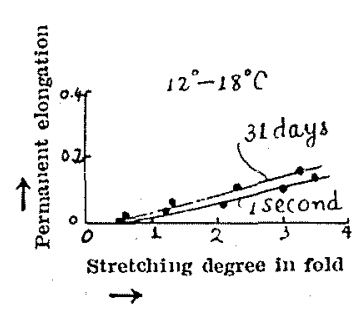

Fig. $4 b$

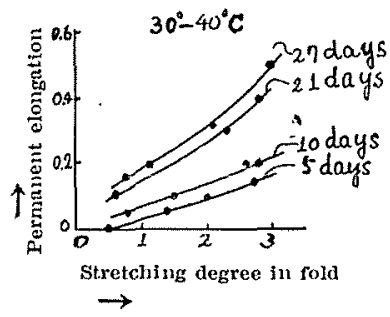

Fig. $4 \mathrm{C}$

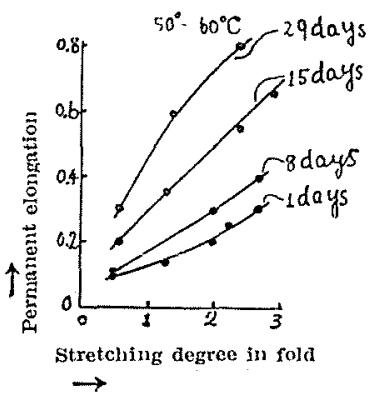

3 , we find that the permanent elongation due to the inter-molecular slipping is very much smaller with vulcanized rubber than with unvulcanized smoked sheet. Though permanent elongation is considerable at high temperatures of $30^{\circ}-40^{\circ} \mathrm{C}$ or $50^{\circ}-60^{\circ} \mathrm{C}$, it is almost unnoticeable at room temperature $\left(12^{\circ}-18^{\circ} \mathrm{C}\right)$ even with the stretching duration of $3 \mathrm{I}$ days; and there is hardly any difference detectable between the stretching of 31 days and that of only a second. This is one of the valuable properties of vulcanized rubber, and it seems to be caused by the hindrance of inter-molecular slipping by virtue of the strong side linkage between neighbouring chain molecules of the rubber, due to the presence of the sulphur molecules.

When unstretched rubber is cooled suddenly to such a low temperature as $-30^{\circ} \mathrm{C}$, the crystallization is suspended and it hardens as amorphous solid by being super-cooled as stated above. It is also stated previously, that the tensile strengths of the smoked sheet rubber and the vulcanized rubber which are cooled suddenly in unstretched state are remarkably low when compared with that cooled in sufficiently stretched state. This is evidently related to the super-cooling. The super-cooling becomes conspicuous and consequently the degree of crystallization gets unnoticeable with decrease of the cooling temperature. In the super-cooled state the intermolecular slipping is much easier to take place by virtue of the lack of some kind of the hooking effect between neighbouring molecules which is remarkable in the crystallized state. The fact that the tensile strength of the rubber ribbons 
which are cooled suddenly in unstretched state decreases from $-20^{\circ} \mathrm{C}$ to about $-70^{\circ} \mathrm{C}$ is thus clearly understood by the increase of the degree of the super-cooling with decrease of the temperature. When the cooling temperature is still lowered to that of liquid air the tensile strenth increases slightly. By the sudden cooling to this low temperature the supercooling will be almost perfect, and a slight increase of the tensile strength at this temperature seems to be caused by the increased difficulty of the inter-molecular slipping due to the increased side cohesive force between neighbouring chain molecules.

The tensile strengths of the vulcanized rubber and smoked sheet rubber which are kept cooled in fully stretched state increase with decrease of the cooling temperature, that of the vulcanized rubber $\mathrm{C}$ being generally much higher than those of the other kinds. This superior tensile strength of the vulcanized rubber $C$ can be explained by the interfering action of sulphur molecules upon inter-molecular slipping by virtue of the enhanced side linkage between neighbouring chain molecules; and the increase of the tensile strengths of the vulcanized rubber and the smoked sheet rubber with decrease of temperature can be ascribed to the increased difficulty of the inter-molecular slipping due to the increased degree of crystallization and of intermolecular sicle cohesion at low temperatures. The tensile strengths of the vulcanized rubber $\mathrm{C}$ and other kinds, which are considerably different at higher temperatures, converge almost to the same value at the temperature of liquid air. This point is especially noteworthy, and it seems to indicate that the tensile strengths of all the vulcanized and the smoked sheet rubbers at this low temperature are mainly related to the force which is necessary to tear the chain molecules of rubber; for, if they were due still considerably to the inter-molecular slipping, the remarkable differences among their values present at higher temperatures should also be present at the temperature of liquid. air. Further, as is seen in Fig. 2, the tensile strength of the vulcanized rubber $\mathrm{C}$ attains at the temperature of dry ice almost to its maximum value assumed at the temperature of liquid air. This seems to indicate that the force necessary to tear a chain molecule of rubber is not much affected by the temperature.

Under the considerations made in the above way, the force necessary to tear the chain molecule of rubber was calculated by regarding the tensile strength of rubber at the temperature of liquid air as exclusively due to this force. It is known that when rubber is crys- 
tallized by stretching the chain molecules of it are arranged parallel to the stretching direction, and that 4 such chain molecules pass through its unit crystal having the cross-sectional area of $8.54 \times 12.65$ $=108 \mathrm{~A}^{2}$ in the direction perpendicular to the stretching direction. Thus if we assume that all rubber molecules in a rubber ribbon which is fully stretched and cooled to the temperature of liquid air are approximately fitted in the crystal lattice, the total number of chain molecules penetrating the cross-sectional area of $\mathrm{I} \mathrm{cm}^{2}$ will be $(4 / \mathrm{r} .08) \times$ $10^{14}=3.7 \times 10^{14}$. Moreover if we take the tensile strength of the fully stretched rubber ribbon at the temperature of liquid air as $1850 \mathrm{Kg} / \mathrm{cm}^{2}$, the force necessary to tear a chain rubber molecule becomes ( $1.85^{\circ} \times$ $\left.10^{6}\right) /\left(3.7 \times 10^{14}\right)=5 \times 10^{-9} \mathrm{~g}$, which may be called " the tensile strength of the chain rubber molecule."

It is known that the decomposition of rubber into isoprene $\left(\mathrm{C}_{5} \mathrm{H}_{\mathrm{S}}\right)$, dipentene $\left(\mathrm{C}_{10} \mathrm{H}_{16}\right)$ and heveene (constitution unknown) begins at the temperature of about $200^{\circ} \mathrm{C}$; and this fact makes it possible to make a rough estimate of the energy necessary to decompose one isoprene group. The manner of thermal motion of each part of the long chain molecule is not of course obvious, but if the mean kinctic energy of an isoprene group is taken as the same with that of a gas molecule at the same temperature, then the simple kinetic theory of gases can be applied to this case. If we represent by $m$ and $\vec{V}^{2}$ the mass and the mean square velocity of a gas molecule, then the average kinetic energy of a molecule at an absolute temperature $T$ is given by

$$
\frac{\mathrm{I}}{2} m \bar{V}^{2}=\frac{3}{2} \frac{R}{N} T,
$$

where $R$ is the gas constant $(R=1.985 \mathrm{cal} /$ degree) and $N$ is the Loschmidt number $\left(N=6 \times 10^{23}\right)$. If we take $T$ as $200^{\circ}+273^{\circ} \mathrm{K}$, at which the decomposition of the rubber molecules into isoprene and others takes place, we get the value of

$$
\frac{\mathrm{x}}{2} m \bar{V}^{3}=9.8 \times 10^{-14} \mathrm{erg}
$$

as the mean kinetic energy of a gas molecule. If this value is taken to be the same with the mean kinetic energy of an isoprene group as a whole at the same temperature, then this can be considered roughly to be the energy required to decompose one isoprene group from the rubber molecule, i. e. the energy needed to tear the chain rubber molecule at the locality of the weakest interatomic cohesion.

The tension acting between the two linking carbon atoms in a 
chain rubber molecule at the locality of the weakest interatomic cohesion will differ according to their distance; and the value of the tensile strength of a chain rubber molecule obtained by the present experiment, can be looked upon as the maximum tension which the two linking carbon atoms can sustain. Thus if we consider that the energy needed to disjoin such carbon linkage is roughly equal to the work done in separating the two linking carbon atoms with the maximum tension for the distance of the order of atomic size, we get for this energy

$$
5 \times 10^{-9} \times 980 \times(\mathrm{r} \sim 2) \times 10^{-8}=5 \times 10^{-14} \sim 10 \times 10^{-14} \mathrm{erg}
$$

which is in good accord with the former estimation.

The above way of estimation of the energy required in breaking the weakest carbon linkage in the chain rubber molecule is very rough of course. However, the agreement between the values estimated in two different ways is satislactory; and it may be looked upon as confirming the value obtained by the present experiment as of the right order for the tensile strength of the chain rubber molecule. The tensile strength of the chain rubber molecule is related to the locality of the weakest interatomic cohesion in it; and it will be naturai to designate the part linked by a single bond between carbon atoms as such a weakest interatomic cohesion. 